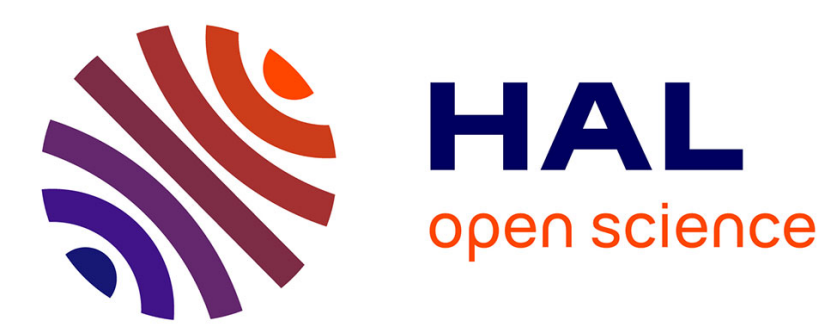

\title{
Effet Mössbauer et techniques complémentaires - I. - La spectroscopie du muon positif
}

\author{
J. Chappert
}

\section{To cite this version:}

J. Chappert. Effet Mössbauer et techniques complémentaires - I. - La spectroscopie du muon positif. Revue de Physique Appliquée, 1980, 15 (6), pp.1063-1071. 10.1051/rphysap:019800015060106300 . jpa-00244824

\section{HAL Id: jpa-00244824 https://hal.science/jpa-00244824}

Submitted on 1 Jan 1980

HAL is a multi-disciplinary open access archive for the deposit and dissemination of scientific research documents, whether they are published or not. The documents may come from teaching and research institutions in France or abroad, or from public or private research centers.
L'archive ouverte pluridisciplinaire HAL, est destinée au dépôt et à la diffusion de documents scientifiques de niveau recherche, publiés ou non, émanant des établissements d'enseignement et de recherche français ou étrangers, des laboratoires publics ou privés. 


\title{
Effet Mössbauer et techniques complémentaires
}

\section{I. - La spectroscopie du muon positif}

\author{
J. Chappert \\ Centre d'Etudes Nucléaires de Grenoble, D.R.F., Laboratoire d'Interactions Hyperfines, \\ 85 X, 38041 Grenoble Cedex, France
}

(Reçu le 14 novembre 1979, révisé le 28 janvier 1980, accepté le 29 janvier 1980)

Résumé. - On présente une revue de la spectroscopie de muon appliquée à la physique de la matière condensée en comparant systématiquement le type d'information obtenu par cette technique et l'effet Mössbauer.

\begin{abstract}
A review is presented of the muon spin rotation technique applied to physics of condensed matter. A systematic comparison is made of the type of information obtained from this technique and from Mössbauer effect.
\end{abstract}

Il peut paraitre curieux qu'une partie importante des travaux de cette réunion de spectroscopistes Mössbauer ${ }^{1}$ ) soit consacrée en fait à d'autres techniques. La raison n'est pas une incitation à délaisser désormais la spectroscopie Mössbauer qui s'est affirmée depuis bien des années comme un outil fondamental de l'étude de la matière condensée et qui a certainement devant elle de longs et beaux jours.

Toutefois si fructueuse soit-elle, une technique a des œillères. Elle ne fournit que des données fragmentaires qui révèlent seulement certaines facettes de la matière étudiée. Ce n'est pas par exemple en multipliant le nombre de spectres Mössbauer que l'on augmente automatiquement la compréhension d'un phénomène physique (là aussi existe un effet de saturation) alors qu'une seule donnée fournie par une autre technique peut se révéler décisive. Il y va donc de la qualité de leur recherche que les spectroscopistes Mössbauer ne se limitent pas à leur technique.

Mais il y va aussi de leur formation permanente en tant que scientifiques. En effet toute technique ne met en jeu qu'un nombre limité de concepts, dont elle exalte d'ailleurs certains aspects aux dépens d'autres, qui sont plus ou moins laissés dans l'ombre. Or il est bien connu que l'on n'assimile parfaitement un concept ou un modèle que lorsque ceux-ci ont été abordés selon des approches différentes et qu'il est

( $\left.{ }^{1}\right)$ Réunion du Groupe Français de Spectrométrie Mössbauer, Poitiers, 31 mai-1er juin 1979. ainsi possible de discerner ce qui est propre aux techniques de ce qui est intrinsèque à la physique.

Nous présenterons donc ici les principes et exemples d'applications de trois techniques qui nous apparait-t-il, en conjonction avec celle-ci. Ce premier bauer, ne serait-ce que par leur caractère microscopique, et qui sont cependant encore peu utilisées, nous apparaît '-il, en conjonction avec celle-ci. Ce premier exposé (I) sera consacré à la spectrošcopie du muon positif ; il sera suivi d'une présentation de l'annihilation de positons (exposé II) et de l'EXAFS, appliqué plus particulièrement aux déterminations structurales de milieux désordonnés (exposé III).

1. Principes de la spectroscopie du muon positif. Dès 1957, on a songé à utiliser le muon comme sonde de la matière condensée. C'est en effet à cette date que Garwin et al. [1], observant l'anisotropie de la désintégration du muon, jetèrent les un de la spectroscopie de muons en suggérant la possible utilisation de cette particule en physique du solide. Nous ne considérerons ici que le muon chargé positivement avec lequel a été obtenu jusqu'à présent l'essentiel des résultats en physique de la matière condensée, alors que le muon négatif trouve plutôt son champ d'application en physique atomique. Le muon positif $\left(\mu^{+}\right)$a deux caractéristiques essentielles : d'une part il teste les sites interstitiels et non substitutionnels comme le font la plupart des sondes des spectroscopies usuelles. D'autre part le $\mu^{+}$n'est généralement pas statique et diffuse dans le solide. En consé- 
quence les quantités mesurées par spectroscopie de muons ( $\mu^{+} \mathrm{SR}$ en anglais pour muon spin rotation) sont toujours une combinaison des propriétés statiques et dynamiques de la sonde $\left(\mu^{+}\right)$et de la matrice (échantillon). C'est ce qui fait à la fois la complexité et la richesse de cette spectroscopie qui, comme nous le verrons, s'apparente à la résonance magnétique nucléaire sur un noyau simple se déplaçant dans le solide [2].

Le $\mu^{+}$a une masse au repos $m_{\mu} c^{2}$ de $106 \mathrm{MeV}$, c'est-à-dire qu'il peut être considéré comme un proton léger $\left(0,11 m_{\mathrm{p}}\right)$. Il a un spin $1 / 2$, comme le proton, et est donc insensible aux effets quadrupolaires (mais nous verrons qu'il peut en provoquer). Sa durée de vie moyenne $\tau_{\mu}$ est de $2,2 \times 10^{-6} \mathrm{~s}$, se désintégrant en un positon et deux neutrinos selon le schéma

$$
\mu^{+} \rightarrow \mathrm{e}^{+}+v_{\mathrm{e}}+\bar{v}_{\mu} .
$$

Deux propriétés jouent un rôle très important :

1) L'émission du positon est anisotrope dans l'espace car elle est corrélée à la direction du spin du $\mu^{+}$. La probabilité d'émission suit une distribution

$$
W(\theta)+1+a \cos \theta
$$

où $\theta$ est l'angle entre les directions du spin du $\mu^{+}$ et du positon, et $a$ est un paramètre voisin de $1 / 3$. Ainsi le positon est émis préférentiellement selon la direction du spin du $\mu^{+}(\theta=0)$.

2) Les faisceaux de $\mu^{+}$(produits à partir d'accélérateur de protons de $\sim 600 \mathrm{MeV}$ ou d'usines à mésons) sont très fortement polarisés $(>80 \%)$ c'est-à-dire que les spins des $\mu^{+}$sont pratiquement tous parallèles entre eux, en l'occurrence antiparallèles à la quantité de mouvement. Connaissant ainsi la polarisation initiale des muons il sera possible d'en suivre l'évolution dans le solide en étudiant l'anisotropie de l'émission des positons.

Le $\mu^{+}$qui pénètre dans la matière a une énergie de l'ordre de 10 à $50 \mathrm{MeV}$. Il est très rapidement thermalisé par diffusion par les électrons en un temps très court $\left(\sim 10^{-9}\right.$ à $\left.10^{-10} \mathrm{~s}\right)$, bien inférieur à son temps de vie. Un fait essentiel est que cette thermalisation ne s'accompagne pas de dépolarisation significative.

L'interaction prédominante entre le $\mu^{+}$et le solide est d'origine électrostatique. C'est elle qui impose au $\mu^{+}$de se maintenir entre les atomes, c'est-à-dire aux sites interstitiels. Mais le $\mu^{+}$possédant un moment magnétique sera aussi sensible aux interactions de type magnétique. S'il voit un champ magnétique $B_{\mu}$, son spin va précesser dans un plan perpendiculaire à $B_{\mu}$ avec la fréquence de Larmor

$$
\omega_{\mu}=\gamma_{\mu} B_{\mu}
$$

où $\gamma_{\mu}$, rapport gyromagnétique du $\mu^{+}$, vaut

$$
8,514 \times 10^{4} \mathrm{rad} \mathrm{s}^{-1} \mathrm{G}^{-1}(0,01355 \mathrm{MHz} / \mathrm{G}) .
$$

Typiquement $\omega_{\mu}$ est de l'ordre de quelques $\mathrm{MHz}$ pour des champs de l'ordre du kG. C'est cette interaction magnétique qui est utilisée pour étudier les propriétés du solide dans lequel est implanté le $\mu^{+}$. En effet grâce à une analyse différentielle en temps, on enregistre dans une direction donnée (Fig. 1a) le taux de positons détectés en fonction du temps $t$ écoulé depuis l'entrée des $\mu^{+}$dans le solide. La courbe classique de décroissance exponentielle (observée en l'absence de perturbation extérieure sur le $\mu^{+}$) est modulée à la fréquence $\omega_{\mu}$. Une telle courbe expérimentale est représentée sur la figure $1 b$. En plus de cette modulation, on observe un amortissement plus ou moins rapide de l'amplitude des oscillations. Cet amortissement reflète un déphasage progressif des spins des muons, résultant d'une certaine distribution des fréquences $\omega_{\mu}$ dont nous exposerons plus loin l'origine physique. La courbe expérimentale de la figure $1 b$ peut être paramétrisée par la fonction

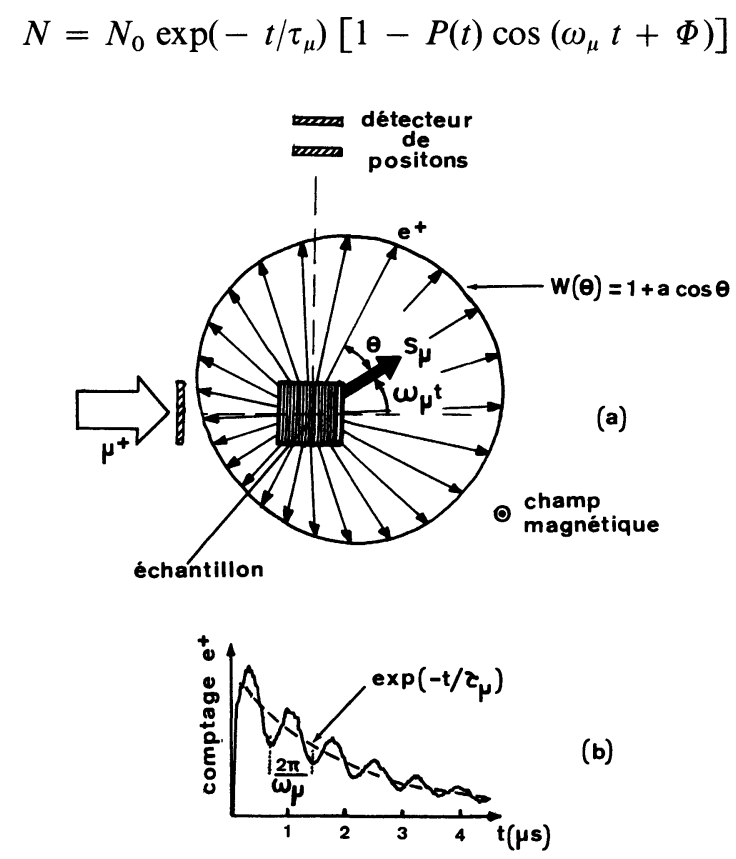

Fig. 1. - Principe d'une expérience de spectroscopie de muons. a) Le champ magnétique, perpendiculaire à la polarisation initiale du faisceau, fait tourner les spins $S_{\mu}$ à la fréquence $\omega_{\mu} . b$ ) Il en résulte une modulation du taux de comptage des positons détectés.

[Principle of $\mu$ SR technique. a) The magnetic field, perpendicular to the initial polarization of the beam, produces a precession of the spins $\mathrm{S}_{\mu}$ with a frequency $\omega_{\mu} . b$ ) As a result a modulation of the positron counting rate is observed.]

où $P(t)$ représente l'évolution de la polarisation avec le temps et s'écrit

$$
P(t)=P(0) \exp \left(-t / T_{2}\right)
$$

$P(0)$ étant la polarisation initiale et $T_{2}$ le temps de relaxation transverse relié à la distribution $\Delta B_{\mu}$ des champs $B_{\mu}$ par la relation

$$
\frac{1}{T_{2}}=\frac{1}{2} \gamma_{\mu} \Delta B_{\mu} .
$$


Ainsi la spectroscopie de $\mu^{+}$permet de mesurer d'une part les champs magnétiques locaux vus par le $\mu^{+}$: cela concerne essentiellement les spécialistes du magnétisme [3] et d'autre part les distributions de champs, liées à une diffusion du $\mu^{+}$de site en site : Tes chercheurs intéressés par des problèmes de diffusion, notamment de l'hydrogène, considèrent le $\mu^{+}$ comme un proton léger et ont ainsi à leur disposition un nouvel isotope de l'hydrogène [4]. L'exposé de ces deux types d'applications et leur complémentarité avec des études Mössbauer, constituera l'essentiel des deux sections suivantes.

2. Diffusion du muon positif. - La très grande mobilité de l'hydrogène dans les métaux, comparée à celle d'autres atomes interstitiels, constitue depuis longtemps une énigme tant pour les chercheurs que pour les industriels pour qui ce problème est étroitement lié à celui du stockage de l'hydrogène utilisé comme source d'énergie. Pressentant que la masse de l'hydrogène jouait un rôle important dans les mécanismes de diffusion, les chercheurs ont étudié la diffusion des différents noyaux : proton, deuton $\left(2 m_{\mathrm{p}}\right)$ et triton $\left(3 m_{\mathrm{p}}\right)$. Avec le muon considéré comme un proton léger $\left(\sim 0,1 m_{\mathrm{p}}\right)$, ils pouvaient ainsi étendre grandement l'éventail des masses de la particule diffusante ce qui explique l'attrait considérable qu'a exercé dès le début la technique $\mu^{+}$SR (il y a quelques années). C'est aussi la raison pour laquelle c'est dans ce domaine que le plus de résultats ont été accumulés, même si à l'heure actuelle de nombreuses questions n'ont pas encore reçu de réponse [4].

Comment mettre en évidence la diffusion du $\mu^{+}$? Il faut pour cela disposer de jalons dans le solide. Ceux-ci seront constitués par les moments magnétiques portés soit par les noyaux (moments nucléaires) soit par les atomes (moments électroniques dans les matériaux magnétiques). Le $\mu^{+}$pourra en effet y être repéré grâce à l'interaction dipolaire entre son moment magnétique $\mu_{\mu}$ et les moments magnétiques environnants $\mu_{\mathrm{i}}$ distants de $r_{\mathrm{i}}$

$$
\mathscr{H}_{\mathrm{dip}}=\frac{\mu_{0}}{4 \pi} \sum_{\mathrm{i}}\left[\frac{\boldsymbol{\mu}_{\mu} \cdot \boldsymbol{\mu}_{\mathrm{i}}}{r_{\mathrm{i}}^{3}}-\frac{3\left(\boldsymbol{\mu}_{\mu} \cdot \mathbf{r}_{\mathrm{i}}\right)\left(\boldsymbol{\mu}_{\mathrm{i}} \cdot \mathbf{r}_{\mathrm{i}}\right)}{r_{\mathrm{i}}^{5}}\right] \text {. }
$$

Le premier métal ainsi étudié a été le cuivre [5]. Celui-ci est soumis à un champ magnétique d'environ $100 \mathrm{G}$ perpendiculaire à la polarisation $P(0)$. Ce champ magnétique $B_{\text {ext }}$ entraîne les spins des muons dans un mouvement de précession (dans un plan perpendiculaire à $B_{\text {ext }}$ ), avec la fréquence $\omega_{\mu}(2)$. Mais $B_{\mu}$, le champ $v u$ par le $\mu^{+}$, n'est pas exactement le champ appliqué $B_{\text {ext }}$. En effet à $B_{\text {ext }}$ il faut ajouter la contribution dipolaire des moments magnétiques des noyaux de cuivre. Ces moments nucléaires, orientés au hasard (aux températures usuelles), créent un champ aléatoire de l'ordre du Gauss qui se superpose à $B_{\text {ext }}$. Il en résulte un spectre de fréquences $\omega_{\mu}$ qui provoque un déphasage plus ou moins rapide des spins. C'est ce que traduisent les courbes expérimentales (Fig. $2 a$ ) où l'on observe à basse température un amortissement des oscillations qui disparaît à haute température, le changement de régime se produisant vers $80 \mathrm{~K}$. Ceci a été interprété en supposant que le
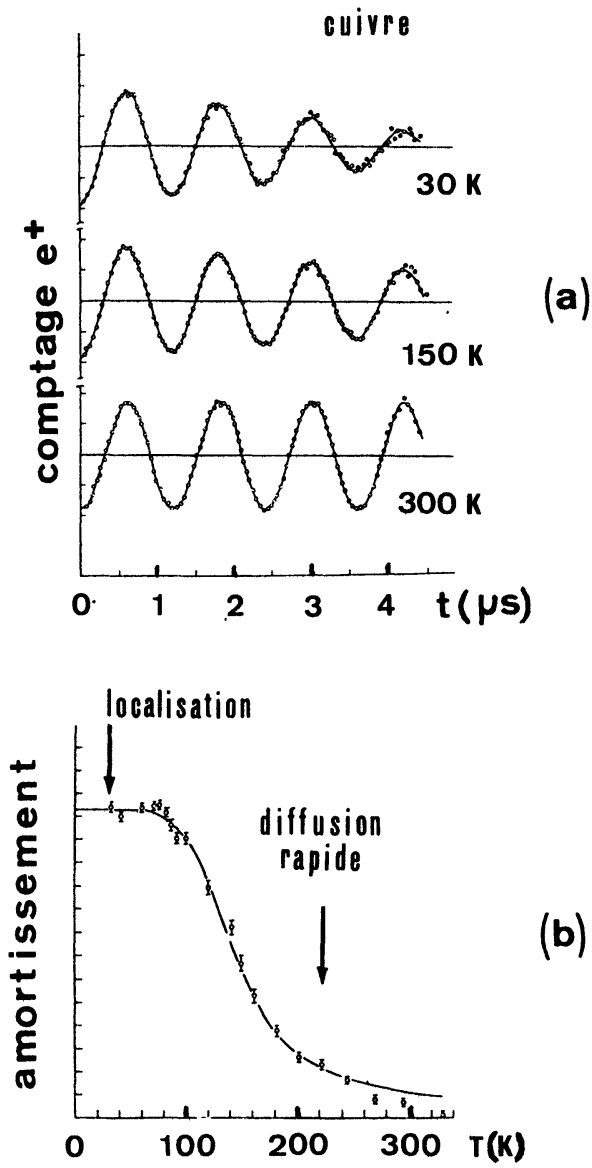

Fig. 2. - a) Oscillations observées dans le cuivre [5] à différentes températures après correction du terme $\exp \left(-t / \tau_{\mu}\right)$. b) Amortissement en fonction de la température [5]. Le terme localisation signifie soit que le $\mu^{+}$est statique soit que sa diffusion est suffisamment lente au cours de sa vie pour qu'elle soit limitée au voisinage d'un site.

[a) Positron counting rate observed in copper [5] at various temperatures after correction for the exponential term $\exp \left(-t / \tau_{\mu}\right) . b$ ) Damping as a function of the temperature [5]. Localization means that the $\mu^{+}$is either static or that its diffusion is slow enough during its lifetime so that it remains in the neigbourhood of one site.]

$\mu^{+}$est localisé à basse température : chaque $\mu^{+}$voit un champ dipolaire légèrement différent, donc la dépolarisation du faisceau est forte c'est-à-dire que les spins se déphasent. A haute température le $\mu^{+}$diffuse rapidement, c'est-à-dire qu'au cours de sa durée de vie $\tau_{\mu}$ il échantillonne un grand nombre de sites (plusieurs milliers). Il ne voit donc finalement qu'un champ dipolaire moyen. Par conséquent la dépolarisation, et par-là même l'amortissement des oscillations, sont beaucoup plus faibles (Fig. 2b). Ceci est tout à fait semblable au phénomène de rétrécissement par le mouvement bien connu en RMN. 
Abragam [6] a montré que la polarisation évoluait avec le temps comme :

$$
P(t)=P(0) \exp \left[-2 \sigma^{2} \tau_{\mathrm{c}} \exp \left(-t / \tau_{\mathrm{c}}\right)-1+1 / \tau_{\mathrm{c}}\right]
$$

où $\tau_{\mathrm{c}}$ est un temps de corrélation lié au temps de résidence du $\mu^{+}$en un site et $\sigma^{2}$ est le second moment de la distribution de champ dipolaire calculé par Van Vleck [7]. Dans la limite $\tau_{\mathrm{c}} \ll \tau_{\mu}\left(\mu^{+}\right.$statique, basse température), la dépolarisation est forte et :

$$
P(t) \sim P(0) \exp \left(-\sigma^{2} t^{2}\right) .
$$

Si $\tau_{c} \ll \tau_{\mu}\left(\mu^{+}\right.$diffusant rapidement, haute température)

$$
P(t) \sim P(0) \exp \left(-2 \sigma^{2} \tau_{\mathrm{c}} t\right) .
$$

La dépolarisation est faible il y a rétrécissement par le mouvement.

Le phénomène de rétrécissement par le mouvement n'est pas non plus inconnu en spectròscopie Mössbauer, qui est aussi un outil pour les études de diffusion [8]. On sait qu'en fait deux situations peuvent s'y rencontrer. Si c'est le noyau Mössbauer qui diffuse, on observe un élargissement de la raie lorsque le temps de saut est comparable au temps de vie nucléaire. C'est ce qui a été observé pour la diffusion de ${ }^{57} \mathrm{Fe}$ dans l'or [9]. Par contre la situation inverse se rencontre lorsque l'atome diffuseur n'est pas l'isotope Mössbauer. Considérons en effet la diffusion d'une impureté interstitielle dans un métal. Lorsque cette impureté se trouve au voisinage de l'isotope Mössbauer, l'abaissement de symétrie qui en résulte se traduit par un changement du déplacement isomérique et par l'apparition d'une interaction quadrupolaire supplémentaire qui élargissent les raies. Mais si cette impureté saute de site en site à une fréquence très grande, l'isotope Mössbauer ne voit plus qu'une interaction moyenne : la raie Mössbauer s'amincit. On retrouve l'effet de rétrécissement par le mouvement. Ceci a par exemple été observé pour la diffusion de l'hydrogène interstitiel dans le tantale (Fig. 3) [10].

Sans entrer dans les détails, mentionnons que la connaissance de la variation thermique du taux de dépolarisation du $\mu^{+}$, est une donnée précieuse pour la mise en évidence de différents régimes de diffusion du $\mu^{+}$, et par analogie de l'hydrogène, [11] dans des gammes de températures et de concentrations jamais atteintes jusqu'ici.

2.1 DÉTERMINATION DU SITE OCCUPÉ PAR LE MUON POSITIF. - Ainsi nous venons de voir que la diffusion du $\mu^{+}$dans les métaux pouvait être mise en évidence grâce à l'interaction dipolaire entre le moment magnétique du $\mu^{+}$et ceux des noyaux du métal. Mais il est possible en outre de préciser le type de site occupé par le $\mu^{+}$. Reprenons l'exemple du cuivre qui comporte des sites interstitiels de types octaédrique et tétraédrique. Si l'on calcule les champs dipolaires vus par le $\mu^{+}$

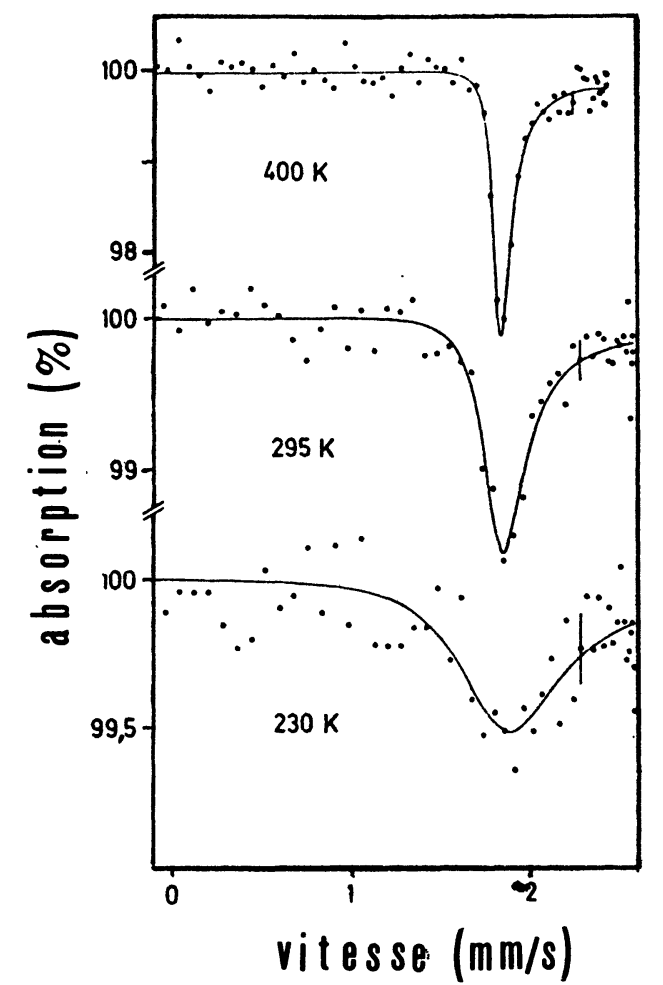

Fig. 3. - Spectres Mössbauer de ${ }^{181} \mathrm{Ta}$ à différentes températures dans une feuille de tantale métallique chargé d'hydrogène $(\mathrm{H} / \mathrm{Ta}=0,018)[10] . \mathrm{A} 400 \mathrm{~K}$ le spectre est identique à celui d'une feuille de tantale pur.

[Mössbauer spectra of ${ }^{181} \mathrm{Ta}$ in a hydrogen-loaded foil of Ta $(\mathrm{H} / \mathrm{Ta}=0.018)[10]$ at various temperatures. At $400 \mathrm{~K}$ the spectrum is identical to that of a pure tantalum foil.]

lorsque le champ $B_{\text {ext }}$ est appliqué selon les directions cristallographiques [100], [110] ou [111], on trouve des valeurs nettement différentes pour le taux d'amortissement $\sigma$ pour les deux types de sites interstitiels (Tableau I). Il est donc possible de déduire le site occupé par le $\mu^{+}$en mesurant ce taux pour diverses orientations de cristal. Une remarque toutefois s'impose ici. Les valeurs du tableau I sont obtenues en supposant que le champ magnétique $B_{\text {ext }}$ définit l'axe de quantification pour le calcul de $\sigma$. Or les expériences sur le cuivre de Camani et al. [12] interprétées par Hartmann [13] ont montré que ceci n'était réalisé que lorsque $B_{\text {ext }}$ était suffisamment fort ( $>5 \mathrm{kG}$ ). En effet, le $\mu^{+}$déforme le réseau cristallin autour de lui et crée un gradient de champ électrique que ressentent les noyaux de cuivre $(I=3 / 2)$. Alors l'axe de quantification n'est plus $B_{\text {ext }}$ et les champs dipolaires doivent

Tableau I. - Taux d'amortissement $\sigma\left(e n \mu \mathrm{s}^{-1}\right)$ calculés pour des muons positifs localisés en site octaédrique ou tétraédrique dans le cuivre [13].

$$
\text { Site Direction du champ }
$$

$\begin{array}{lccc}- & {[100]} & {[111]} & {[110]} \\ \text { Octaédrique } & - & - & - \\ \text { Tétraédrique } & 0,307 & 0,067 & 0,164 \\ & 0,077 & 0,319 & 0,279\end{array}$


être calculés en considérant le ćas d'interactions quadrupolaires et magnétiques combinées. Les calculs de Mathias et al. [14], bien connus des spectroscopistes Mössbauer, sont utilisés à cet effet. Dans le cas du cuivre, les valeurs du tableau I ne sont observées que lorsque le rapport des interactions magnétiques et quadrupolaires est supérieur à environ 10 (Fig. 4). $\mathrm{La}$ comparaison avec les données expérimentales montre de façon claire que le $\mu^{+}$occupe dans le cuivre les sites interstitiels de symétrie octaédrique.

Il est intéressant de rappeler que c'est ce qui est précisément observé pour la localisation de l'hydrogène dans le cuivre, confirmant que le muon positif peut être considéré comme un isotope léger de l'hydrogène.

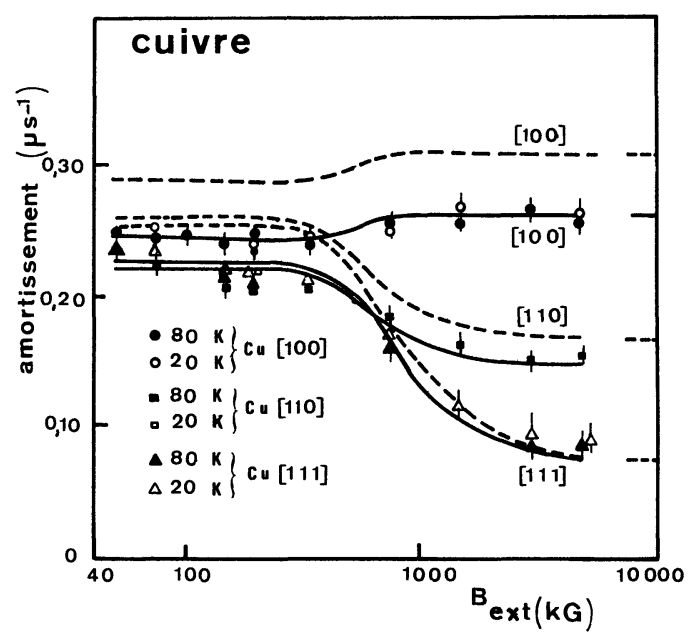

Fig. 4. - Amortissement des oscillations dans un monocristal de cuivre pour diverses orientations par rapport au champ magnétique extérieur $B_{\text {ext }}$ [12]. Les courbes en traits interrompus ont été calculées utilisant le modèle de Hartmann [13] en supposant que le $\mu^{+}$ se localise en site octaédrique. Les courbes continues tiennent compte d'une dilatation du réseau cristallin de $5 \%$. Les lignes à l'extrême droite indiquent le taux d'amortissement en l'absence d'interaction quadrupolaire.

[Damping of the oscillations in a copper single crystal for various orientations with respect to the external magnetic field $B_{\text {ext }}[12]$. Dashed lines are calculated using a model by Hartmann [13] assuming that the $\mu^{+}$is localized in a ortahedral site. Solid lines take into account the $5 \%$ expansion of the crystal lattice. Lines on the right part give the damping rate in the absence of quadrupole interaction.]

2.2 EFFET DES IMPURETÉS SUR LA DIFFUSION DU MUON POSITIF. - Les premières études de diffusion $\mathrm{du} \mu^{+}$dans les métaux ont fourni des données souvent difficiles à interpréter, différents groupes de chercheurs obtenant des résultats dissemblables. Il est apparu petit à petit que le taux d'impuretés jouait un rôle primordial, au point qu'il n'est pas rare que certains échantillons ne donnent aucun signal, c'est-àdire que les muons se dépolarisent presque immédiatement dans le cristal car ils sont piégés par les impuretés. Un exemple remarquable est constitué par le niobium, dont l'étude a été effectuée sur des échantillons contenant des taux contrôlés d'impuretés $[15,16,17]$.

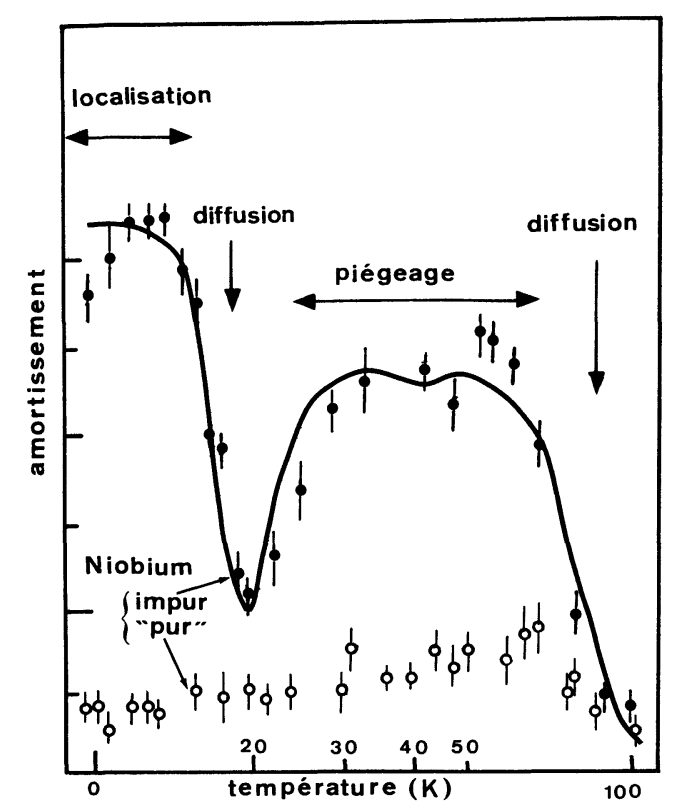

Fig. 5. - Amortissement des oscillations dans deux échantillons de niobium pur et impur en fonction de la température $[15,16]$.

[Thermal variation of the damping of the oscillations in two niobium samples of different purity $[15,16]$.]

La figure 5 montre la variation thermique complexe du taux d'amortissement observé pour un échantillon de niobium contenant 10 à 20 p.p.m. d'impuretés $(\mathrm{N}, \mathrm{C}, \mathrm{O})$ alors qu'une variation monotone est obtenue pour un échantillon pur, renfermant moins de 3 p.p.m. d'impuretés $[15,16]$. La première courbe a été interprétée en termes de piégeage et dépiégeage successifs da muon tandis que la deuxième correspond vraisemblablement à l'apparition d'un régime de diffusion cohérente du $\mu^{+}$, comme dans l'aluminium [11] et le fer [18] ultra-purs à basse température.

Dans ce dernier cas (fer) une étude de la fréquence $\omega_{\mu}$ en fonction de la température et du taux d'impureté de carbone a permis de faire une corrélation intéressante avec des expériences de spectroscopie Mössbauer par Cadeville et al. [19].

3. Champ magnétique interstitiel. - Jusqu'ici nous nous sommes intéressés à $\Delta B_{\mu}$, la distribution de champs vus par le $\mu^{+}$et nous avons montré comment cette distribution pouvait être reliée à la diffusion du $\mu^{+}$par l'intermédiaire du taux d'amortissement. Mais la valeur même de $B_{\mu}$ (déduite de $\omega_{\mu}$ ) est d'une extrême importance car il existe peu de méthodes permettant de sonder les champs magnétiques interstitiels. Dans un composé magnétique soumis à un champ magnétique extérieur, $B_{\text {ext }}, B_{\mu}$ est la somme de plusieurs contributions [3]

$$
\mathbf{B}_{\mu}=\mathbf{B}_{\mathrm{ext}}+\mathbf{B}_{\mathrm{dem}}+\mathbf{B}_{\mathrm{L}}+\mathbf{B}_{\mathrm{dip}}+\mathbf{B}_{\mathrm{hf}}
$$

où $\mathbf{B}_{\mathrm{L}}=\frac{4 \pi}{3} \mathbf{M}_{\mathrm{s}}$ est le champ de Lorentz à l'intérieur d'un domaine, $B_{\text {dip }}$ est le champ dipolaire à l'intérieur 
de la cavité de Lorentz. Pour un échantillon non aimanté avec $B_{\text {ext }}=0$, le champ démagnétisant $B_{\text {dem }}$. est nul. Les trois premières contributions sont aisément accessibles. Nous allons porter notre attention sur les deux derniers termes. Plusieurs remarques s'imposent auparavant :

a) Les valeurs observées pour $B_{\mu}$ (quelques $\mathrm{kG}$ ) sont très petites comparées aux champs mesurés par spectroscopie Mössbauer ou résonance magnétique nucléaire (quelques centaines de $\mathrm{kG}$ ). Par conséquent les termes $B_{\mathrm{L}}$ et $B_{\text {dip }}$ sont loin d'être négligeables. Leur évaluation précise est donc d'une importance extrême en particulier si l'on veut pouvoir en déduire le terme hyperfin $B_{\mathrm{hf}}$.

$b$ ) Le champ hyperfin $B_{\text {hf }}$ est dû uniquement à la polarisation des électrons de conduction à l'emplacement du $\mu^{+}$car ce dernier ne possède pas de cortège électronique. Cette polarisation a une double origine dans les métaux : l'une résultant de l'aimantation locale (métaux magnétiques), l'autre, du type déplacement de Knight, produite par le champ magnétique extérieur. Les déplacements de Knight ont pu être mesurés pour de nombreux métaux non magnétiques [20] avec l'avantage sur la résonance magnétique nucléaire que le $\mu^{+}$teste l'échantillon en volume et non uniquement sa surface : l'effet de peau n'existe pas.

c) Le signe du champ $B_{\mu}$ peut être déterminé à partir de la phase initiale de la précession observée par le détecteur faisant un angle de $90^{\circ}$ avec la polarisation initiale du muon.

Examinons maintenant séparément les deux contributions $B_{\text {dip }}$ et $B_{\mathrm{hf}}$.

3.1 ChAmp Dipolaire - L'interaction dipolaire entre le moment magnétique $\boldsymbol{\mu}_{\mu}$ du muon et les moments magnétiques environnants $\boldsymbol{\mu}_{\mathrm{i}}$ est donnée par l'équation (6). Cette interaction est nulle lorsque le site interstitiel occupé par le $\mu^{+}$a une symétrie cubique. C'est par exemple le cas du nickel [21]. Par contre lorsqu'elle n'est pas nulle, son estimation est importante [22] car elle donne la possibilité de déterminer le site du $\mu^{+}$. Prenons le cas du cobalt étudié en détail par deux groupes [23, 24]. La figure 6 montre les signaux de précession observés à différentes températures. On observe que le champ $B_{\mu}$ décroît d'abord quand la température augmente, s'annule vers $500 \mathrm{~K}$ puis se met à croître (Fig. 7). On suppose que le champ de Lorentz $B_{\mathrm{L}}$ suit la même variation thermique que l'aimantation. Un calcul classique de $B_{\text {dip }}$ peut être effectué connaissant le moment dipolaire du cobalt, les paramètres du réseau, la direction de l'aimantation locale et le site du $\mu^{+}$. Pour ce dernier deux possibilités existent : sites à symétrie octaédrique ou tétraédrique. Le moment dipolaire du cobalt a été pris égal à $1,72 \mu_{\mathrm{B}} \frac{M_{\mathrm{s}}(T)}{M_{\mathrm{s}}(0)}$. L'aimantation locale est connue d'après les données de neutrons, les paramètres du réseau d'après celles de rayons $\mathrm{X}$. Sur la figure 7

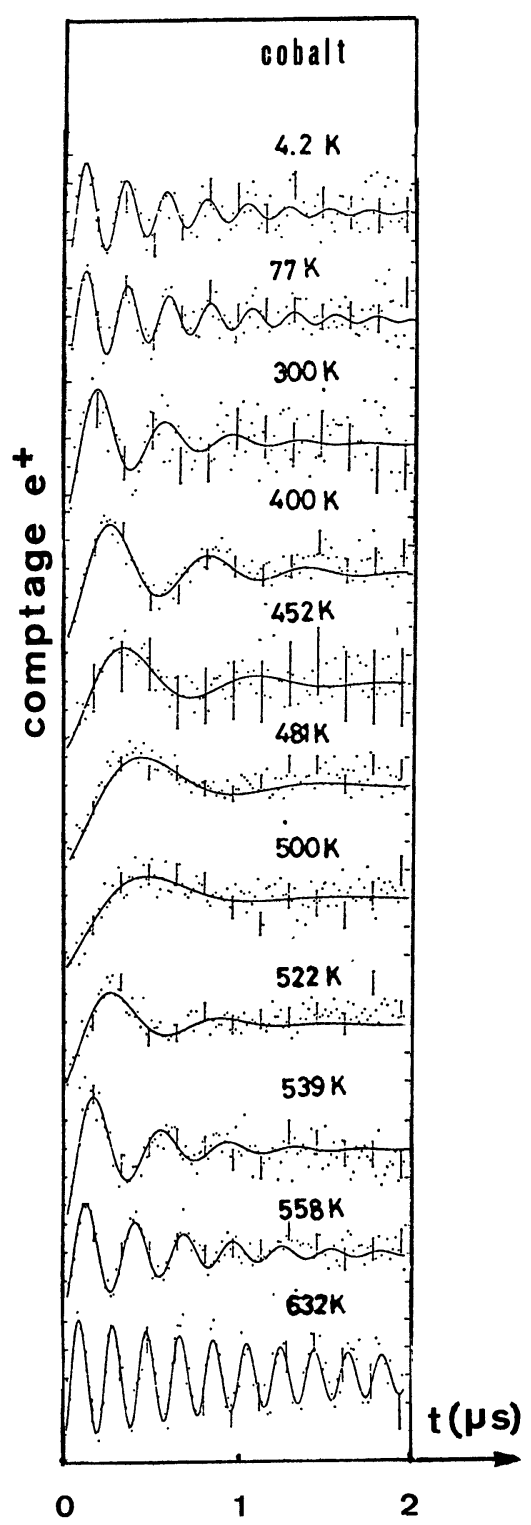

Fig. 6. - Oscillations observées dans le cobalt à différentes températures [24].

[Oscillations observed in metallic cobalt at various temperatures [24].]

sont indiquées les deux variations thermiques attendues pour $H_{\mathrm{dip}}$ (sites octaédrique et tétraédrique). On note dans les deux cas un saut entre $500 \mathrm{~K}$ et $600 \mathrm{~K}$ dû à un changement d'orientation de $90^{\circ}$ de la direction de l'aimantation. A partir de l'équation (10), on déduit $B_{\mathrm{hf}}$ dans chacune des deux hypothèses de sites. On observe que seulement dans le cas d'un site octaédrique, $B_{\mathrm{hf}}$ varie d'une façon monotone en traversant la zone de rotation de spin. Les auteurs en ont conclu que c'était bien ce site qui était occupé par le muon car il n'y a pas de raison pour que $B_{\mathrm{hf}}$ subisse un changement important dans cette zone comme cela serait le cas pour le site tétraédrique.

On voit donc dans cet exemple le rôle important joué par le terme dipolaire, qui pourtant ne vaut ici que $200 \mathrm{G}$ environ. Il est intéressant de rapprocher 


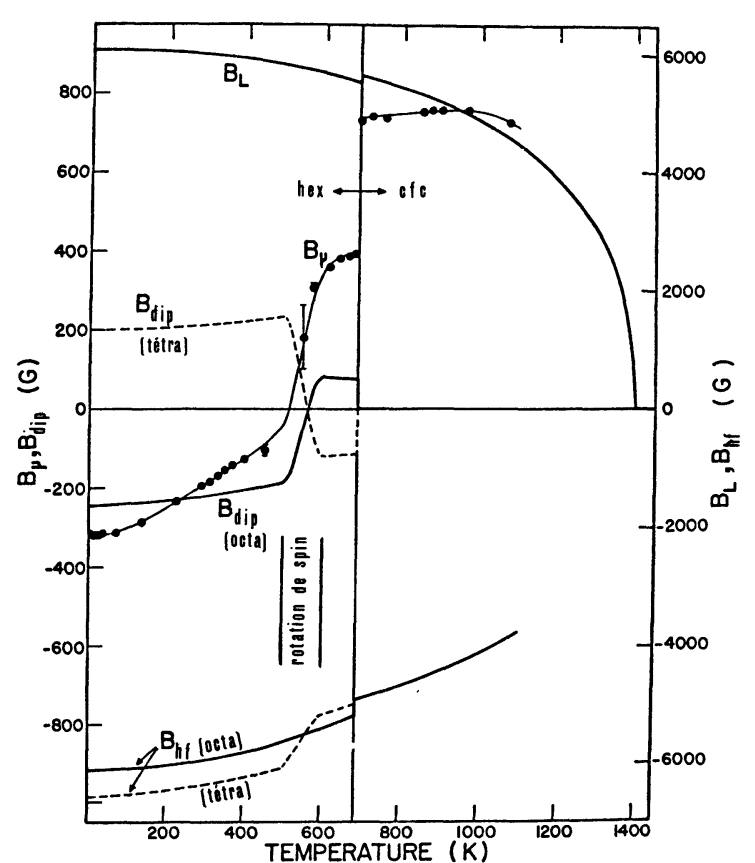

Fig. 7. - Variation en fonction de la température du champ $B_{\mu}$ et du champ $B_{\mathrm{hf}}$ dans le cobalt [23]. Une transition de phase cristallographique (hex $\rightarrow$ c.f.c.) se produit à $690 \mathrm{~K}$.

[Thermal variation of $B_{\mu}$ and $B_{\mathrm{hf}}$ in cobalt [23]. A crystallographic phase transition (hex $\rightarrow$ f.c.c.) takes place at $690 \mathrm{~K}$.]

cette étude de celle de Perlow et al. [25] qui, par spectroscopie Mössbauer de ${ }^{57} \mathrm{Fe}$ dilué dans le cobalt, ont essayé de mettre en évidence l'anisotropie du champ hyperfin dans le cobalt résultant du terme dipolaire. Mais leurs résultats ne permettaient pas de conclure pour deux raisons : au terme dipolaire s'ajoute en spectroscopie Mössbauer une autre contribution anisotrope, le champ orbital. De plus la valeur du champ hyperfin total, $\sim 317 \mathrm{kG}$, rend difficile la mise en évidence de contribution de l'ordre du kilogauss au maximum.

On peut encore citer un exemple bien connu des spectroscopistes Mössbauer, celui de la transition de Morin dans $\alpha-\mathrm{Fe}_{2} \mathrm{O}_{3}$. Un grand nombre d'études Mössbauer lui a été consacré [26]. Si cette transition, qui correspond à une rotation de $90^{\circ}$ de la direction des moments magnétiques (parallèles à l'axe $c$ à $T<263 \mathrm{~K}$, perpendiculaire au-dessus) se voit très nettement en spectroscopie Mössbauer par étude du couplage quadrupolaire au noyau ${ }^{57} \mathrm{Fe}$, elle est beaucoup plus difficile à mettre en évidence à partir de la variation thermique du champ hyperfin à ce même noyau car de nouveau $B_{\text {dip }}$ ne contribue que pour quelques $\mathrm{kG}$ à $B_{\mathrm{hf}}(517 \mathrm{kG})$. Or en $\mu^{+} \mathrm{SR}$ et en l'ab-

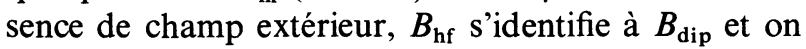
observe, comme pour l'interaction quadrupolaire en spectroscopie Mössbauer, que $B_{\text {dip }}$ change d'un facteur 2 au passage de la transition de Morin (Fig. 8) [27].

Nous avons, dans ce qui précède, supposé implicitement que le champ dipolaire que l'on peut calculer

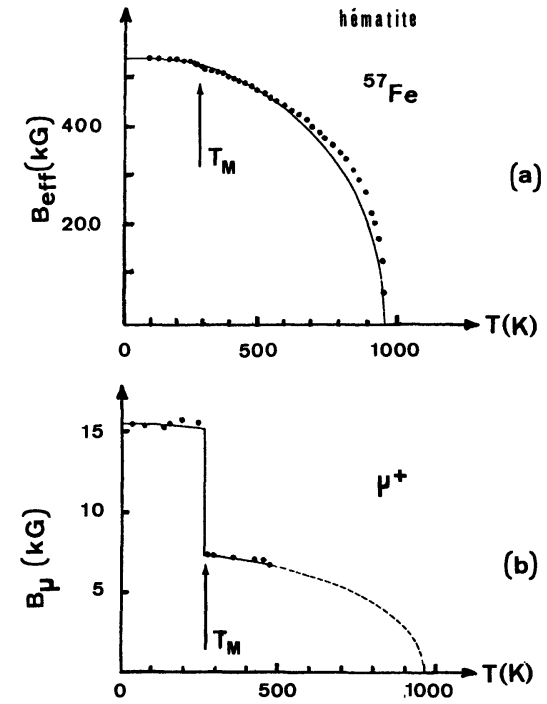

Fig. 8. - Mise en évidence de la transition de Morin $\left(T_{\mathrm{M}}=263 \mathrm{~K}\right)$ dans $\alpha-\mathrm{Fe}_{2} \mathrm{O}_{3}$ par : a) Spectroscopie Mössbauer, champ effectif au noyau ${ }^{57} \mathrm{Fe}[26]$. La ligne continue représente la fonction de Brillouin pour un spin électronique 5/2. b) Spectroscopie de muons positifs, champ vu par le muon [27].

[Evidence for the Morin transition $\left(T_{M}=263 \mathrm{~K}\right)$ in $\alpha-\mathrm{Fe}_{2} \mathrm{O}_{3}$ from : a) Mössbauer spectroscopy, effective field at the ${ }^{57} \mathrm{Fe}$ nucleus [26]. The solid line represents the Brillouin function for an electronic spin $5 / 2 . b) \mu \mathrm{SR}$, field seen by the $\mu^{+}$[27].]

classiquement est celui que voit réellement le $\mu^{+}$. Cependant comme l'ont discuté Nishida et al. [24] plusieurs facteurs sont à même de provoquer un écart entre ces deux valeurs :

a) Du fait de sa faible masse, le $\mu^{+}$présente d'importantes oscillations de point zéro autour de la position d'équilibre. Par conséquent le champ dipolaire réel ressenti par le $\mu^{+}$n'est pas forcément celui existant ponctuellement au centre du site mais plutôt une moyenne spatiale qui tient compte de la taille finie du site occupé par le $\mu^{+}$.

b) Le $\mu^{+}$étant chargé positivement distort le réseau en repoussant les atomes voisins diminuant d'autant leur contribution au champ dipolaire. Cette distorsion correspond dans le cuivre à une dilatation du réseau d'environ $5 \%$.

Il apparaît cependant que ces mécanismes n'introduisent pas une déviation importante de $B_{\text {dip }}$ par rapport à la valeur calculée. Fiory et al. estiment même que les deux effets précédents peuvent s'annuler l'un l'autre.

3.2 ChAMP HYPERFIN. - Le champ hyperfin mesuré par $\mu^{+} \mathrm{SR}, \boldsymbol{B}_{\mathrm{hf}}$, provient de l'interaction de contact (Fermi) entre le moment magnétique du $\mu^{+}$ et les électrons de conduction polarisés par l'aimantation du domaine. Il mesure donc une aimantation locale ou microscopique qu'il est instructif de comparer soit à l'aimantation macroscopique, $M_{\mathrm{s}}$, obtenue par mesures magnétiques, soit à l'aimantation interstitielle, $M_{\text {int }}$, mesurée par neutrons polarisés. 
Tableau II. - Champ hyperfin à saturation, champ hyperfin par magnéton de Bohr et coefficient de renforcement pour les métaux ferromagnétiques : $\mathrm{Fe}, \mathrm{Co}, \mathrm{Ni}[24]$.

$\begin{array}{cccc}\text { Métal } & \begin{array}{c}B_{\mathrm{hf}} \\ (\mathrm{kG})\end{array} & \begin{array}{c}B_{\mathrm{hf}} / \mu_{\mathrm{B}} \\ \left(\mathrm{kG} / \mu_{\mathrm{B}}\right)\end{array} & \propto \\ - & - & - & - \\ \mathrm{Fe} & -11,1 & -5,0 & 10,0 \\ \mathrm{Co} & -6,1 & -3,6 & 3,0 \\ \mathrm{Ni} & - & -1,0 & 1,0\end{array}$

Une telle comparaison, indiquée dans le tableau II pour quelques métaux ferromagnétiques, permet quelques conclusions :

a) Il n'y a pas de relation simple entre $B_{\mathrm{hf}}$ et $M_{\mathrm{s}}$ car $B_{\mathrm{hf}} / \mu_{\mathrm{B}}$ varie entre -1 et -5 .

b) On remarque que $B_{\mathrm{hf}}$ est systématiquement négatif, ce qui est en accord avec le signe également négatif de $M_{\text {int }}$ observé par neutrons polarisés.

c) Il n'y a cependant pas de facteur de proportionnalité constant entre $B_{\mathrm{hf}}$ et $M_{\text {int }}$. Le champ produit par l'aimantation locale en l'absence de muon vaut $\frac{8 \pi}{3} M_{\text {int }}$, mais le $\mu^{+}$ressent en fait un champ $\frac{8 \pi}{3} \propto M_{\text {int }}$, avec $\alpha$ variant entre $1(\mathrm{Ni})$ et $10(\mathrm{Fe}) . \mathrm{Ce}$ renforcement du champ de contact est généralement attribué à l'écran que forment les électrons de conduction autour du $\mu^{+}$pour tenter de neutraliser sa charge positive.

d) Enfin la variation thermique de $B_{\mathrm{hf}}$, normalisé à sa valeur à saturation, est loin de suivre celle de l'aimantation macroscopique (Fig. 9).

Plusieurs causes possibles ont été proposées pour tenter d'expliquer l'ensemble des observations précédentes [3,24] sans qu'il ait été possible d'apprécier

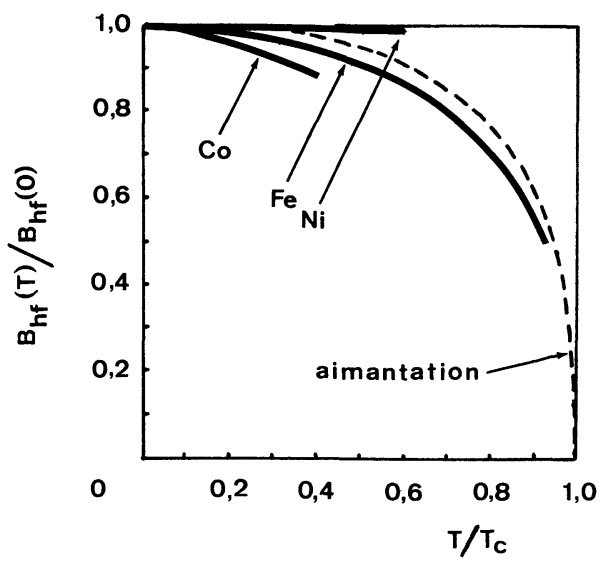

Fig. 9. - Variation thermique du champ hyperfin réduit du muon dans $\mathrm{Fe}, \mathrm{Co}, \mathrm{Ni}$, comparé à l'aimantation macroscopique [24].

[Thermal variation of the reduced hyperfine field in $\mathrm{Fe}, \mathrm{Co}, \mathrm{Ni}$ compared with the macroscopic magnetization [24].]

leur impact de façon quantitative. Ces causes ont en fait été déjà rencontrées tout au long de cet exposé : distorsion du réseau par le muon, effet de moyenne dû à la diffusion du muon, localisation non ponctuelle du muon, écrantage par les électrons de conduction, dilatation thermique affectant différemment $B_{\mathrm{hf}}(T)$ et l'aimantation. Des expériences sous pression sur le nickel et le fer ont par exemple été entreprises sur ce dernier point mais il est difficile de conclure [28], car en fait toutes les causes précédentes agissent vraisemblablement simultanément, ceci même à cause de la présence du muon qui n'est donc pas une sonde innocente. Mais il n'est pas exclu que certains effets reflètent des propriétés intrinsèques des électrons aux sites interstitiels. L'élucidation de toutes ces questions est un beau sujet d'étude pour les théoriciens [29], aidés par les expérimentateurs qui ont devant eux un vaste champ d'investigation encore très inexploré.

\section{Bibliographie}

[1] Garwin, R. L., Lederman, L. M. et Weinrich, M., Phys. Rev. 105 (1957) 1415.

[2] De nombreux articles de revue ont déjà été consacrés à la $\mu^{+}$SR. On pourra consulter en particulier BREWER, J. H. et Crowe, K. M., Ann. Rev. Nucl. Part. Sci. 28 (1978) 239. Pour une mise à jour des plus récents résultats, voir le compte rendu du premier Colloque International sur la spectroscopie de muons dans la revue Hyperfine Interactions vol. 6 (1978)

[3] Yamazaki, T., Physica 86-88B (1977) 1053.

SCHENCK, A., Hyp. Inter. 4 (1978) 282.

[4] Seeger, A., dans Hydrogen in Metals I, édité par Alefeld G. et Wölkl, J. (Springer Verlag, Berlin) 1978.

[5] Gurevich, I. I., Meleshko, E. A., Muratova, I. A., Nikolsky, B. A., Roganov, V. S., Selivanov, V. I., Sokolov, B. V., Phys. Lett. 40A (1972) 143.

GrebinNik, V. G., GuRevich, I. I., Zhukov, V. A., Manich, A. P., Meleshro, E. A., Muratova, I. A., Nikolsky, B. A., Selivanov, V. I. et Suetin, V. A., Sov. Phys. J.E.T.P. 41 (1976) 777.
[6] Abragam, A., The principles of nuclear magnetism (Oxford, University Press, Londres), 1971.

[7] VAN VleCK, J. H., Phys. Rev. 74 (1948) 1168.

[8] JANOT, C., J. Physique 37 (1976) 253.

[9] Mullen, J. G. et Knauer, R. C., dans Mössbauer Effect Methodology vol. 5, édité par Gruverman I. J. (Plenum Press, New York) 1970.

[10] Heidemann, A., Kaindl, G., Salomon, D., Wipf, H. et Wortmann, G., Phys. Rev. Lett. 36 (1976) 213.

[11] Hartmann, O., Karlsson, E., Norlin, L. O., Richter, D. et Ninikoski, T. O., Phys. Rev. Lett. 41 (1978) 1055.

[12] Camani, M., Gygax, F. N., Ruegg, W., Schenck, A. et Schilling, H., Phys. Rev. Lett. 39 (1977) 836.

[13] Hartmann, O., Phys. Rev. Lett. 39 (1977) 832.

[14] Matthias, E., Schneider, W. et Steffen, R. M., Phys. Rev. 125 (1962) 261.

[15] Borghini, M., Ninikoski, T. O., Soulie, J. C., Hartmann, O., Karlsson, E., Norlin, L. O., Pernestal, K., Kehr, K. W., Richter, D. et Walker, E., Phys. Rev. Lett. 40 (1978) 1723. 
[16] Ninikoski, T. O., Hartmann, O., Karlsson, E., Norlin, L. O., Pernestal, K., Kehr, K. W., Richter, D., Walker, E. et Schulze, K., Hyp. Inter. 6 (1978) 229.

[17] Birnbaum, H. K., Camani, M., Fiory, A. T., GygaX, F. N., Kossler, W. J., RuegG, W., SChenck, A. et Schilling, H., Phys. Lett. 65A (1978) 435.

[18] Yaouanc, A., Dufresne, J. F., Longobardi, R., Pezetti, J. P., Chappert, J., Hartmann, O., Karlsson, E. et NorLIN, L. O., J. Phys. F 9 (1979) 2157.

[19] Cadeville, M. C., Friedt, J. M. et Lerner, C., J. Phys. F 7 (1977) 123.

[20] Camani, M., Gygax, F. N., Ruegg, W., Schenck, A., Schilling, H., Klempt, E., Schulze, R. et Wolf, H., Phys. Rev. Lett. 42 (1979) 679.

[21] Patterson, B. D. et Falicov, L. M., Solid State Commun. 15 (1974) 1509.

Nagamine, K., Nagamiya, S., Hashimoto, O., Nishida, N. et YamazaKI, T., Hyp. Inter. 1 (1976) 517.

[22] Kronmüller, H., Hilzinger, H. R., Monachesi, P. et SegGer, P., Appl. Phys. 18 (1979) 183.

[23] Graf, H., Kündig, W., Patterson, B. D., Reichart, W., Roggwiller, P., Camani, M., Gygax, F. N., RüegG, W Schenck, A. et Schilling, H., Phys. Rev. Lett. 37 (1976) 24.

[24] Nishida, N., Nagamine, K., Hayano, R. S., Yamazaki, T., Fleming, D. G., Duncan, R. A., Brewer, J. H., AhKtar, A., Yasuoka, H., J. Phys. Soc. Japan 44 (1978) 1131.
[25] Perlow, G. J., Johnson, C. E. et Marshall, W., Phys. Rev. 140 (1965) A875.

[26] Ce n'est pas possible de citer toutes les études Mössbauer sur $\alpha-\mathrm{Fe}_{2} \mathrm{O}_{3}$. Voir par exemple Ono, K. et ITO, A., J. Phys. Soc. Japan 17 (1962) 1012;

Imbert, P. et Gerard, A., C.R. Hebd. Sean. Acad. Sci. 257 (1963) 1054 ;

VAN Der Woude, F., Phys. Status Solidi 17 (1966) 417;

Ruskov, T., Tomov, T. et Georgiev, S., Phys. Status Solidi A 37 (1976) 295.

[27] Graf, H., Hofmann, W., Kündig, W., Meier, P. F., PatTerson, B. D. et Rodrigez, A., Solid State Commun. 25 (1978) 1079.

De nouveaux résultats du même groupe (Hyp. Inter. 6 (1978) 99) montrent qu'il existe en fait deux fréquences à basse température mais l'essentiel des observations concernant la rotation de spin reste valable.

[28] Butz, T., Chappert, J., Dufresne, J. F., Hartmann, O., Karlsson, E., Lindgren, B., Longobardi, R., Norlin, L. O., Pezetti, J. P. et Yaouanc, A., Communication à la Conférence Internationale de Magnétisme, Munich, Sept. 1979.

[29] Pour une revue des différents modèles proposés voir RAO, G. N. et Singhwi, A. K., Phys. Status Solidi B 84 (1977) 9 et les nombreux articles dans Hyp. Inter. vol. 6 (1978). 